\title{
Routine outcome measurement: recovery, quality of life and co-production ${ }^{\dagger}$
}

\author{
Jed Boardman
}

\section{Summary}

Patient-reported outcome measures (PROMs) are self-rated, but may not take in other aspects of the patient's perspective, such as the inclusion of domains that reflect service-user priorities. The clinician's view still has priority, although this situation has shifted in recent years. The Recovering Quality of Life (ReQoL) offers an advance in this area.

\section{Declaration of interest}

None.

\section{Copyright and usage}

(c) The Royal College of Psychiatrists 2018.
Jed Boardman is visiting Senior Lecturer in Social Psychiatry at the Institute of Psychiatry, Psychology and Neuroscience and Senior Policy Adviser at the Centre for Mental Health, London. His interests include aspects of social and community psychiatry, employment and personal recovery.

The health service policies of successive UK governments have focused on delivering improved patient outcomes and the 'patients' own assessment of their health and health-related quality of life.' ${ }^{1}$ These policy decisions have led to an increased interest in measuring patient-reported outcome measures (PROMs) and their routine use began in England in 2009. ${ }^{1}$ PROMs are viewed as structured questionnaires that 'ask patients about their health from their point of view ${ }^{1}$ and such patient-rated inventories are common in all forms of health research. Nevertheless, in the UK National Health Service, the need to provide these routine measures raises several matters, including what constitutes the 'patients point of view', what domains are measured and their practical implementation.

\section{Service-user participation}

A common view of PROMS is that devising a symptom questionnaire that is rated by the service user is sufficient to capture their 'point of view'. But this approach will not necessarily capture the aspects of health that people consider important, nor their relative value. In a previous editorial in the British Journal of Psychiatry, Rachel Perkins ${ }^{2}$ examined the relative priority of service-user and clinician views of mental health services, concluding that outcome measures need to be designed in collaboration with patients and noted that 'surely it is time both systematically to ascertain users' goals and accord them the status currently enjoyed by the priorities of clinicians?'. ${ }^{2}$ Has anything changed in the past 16 years? The emphasis on the patients' view and involvement in outcome design is not new, but has been more recently stressed in physical medicine ${ }^{3}$ and in research studies. ${ }^{4}$ Generally, the patients' perspective has been neglected in the evaluation of treatment outcomes for mental health conditions, but this may be changing. Trujols et $a l,{ }^{5}$ accepting that PROMs should be self-rated, classified them according to whether they are developed by patients; developed with patient participation; developed to reflect patient values; or are patient-irrelevant. Patient-generated measures

† See pp. 42-49, this issue. have been successfully developed and used $^{6}$ and research funders increasingly specify that service users are involved in the design and execution of research projects. ${ }^{7,8}$ The Recovering Quality of Life (ReQoL), in this issue, is an example of a patient-centred/ patient-reported outcome measure. ${ }^{9}$

Involving service users in their own care and in research practice has been increasingly stressed in recent years, evolving from a passive role in participation to a more active role incorporating co-production. Service users are increasingly involved in mental health research and in the UK the service-user research workforce is diverse, mature and highly skilled. ${ }^{7}$ This involvement not only gives personal benefits to those involved, but improves the recruitment rates in the associated studies. ${ }^{8}$

The development of outcome measures with patient input is consistent with bioethical principles and the development of improved content validity. The use of PROMS may improve communication and decision-making between clinicians and patients, and satisfaction with care. ${ }^{2}$

\section{What domains to assess?}

Using purely symptom domains for a mental health PROM is insufficient to capture the wider range of outcomes valued by service users. ${ }^{5}$ Commonly identified from personal accounts of service users' own recovery are factors relating to hope, control and opportunity and a recent systematic review summarised these under the acronym CHIME (Connectedness, Hope, Identity, Meaning, Empowerment). ${ }^{10}$ These factors are consistent with the definition of personal recovery, which relates to the process through which people find ways of living meaningful lives with or without ongoing symptoms of their condition. A review of current measures of personal recovery, identified 13 possible PROMS, one of which (the Questionnaire about the Process of Recovery - QPR) covers the CHIME framework and none of which demonstrated all the psychometric properties evaluated. ${ }^{11}$ None of the 13 measures could be unequivocally recommended by the reviewers. It seems that although there are many existing measures of personal recovery, many of which are personcentred, none yet meet the rigorous standards for psychometric quality.

The ReQoL casts a new light on the development of a recoveryorientated PROM. ${ }^{9}$ It is based on previous work examining the quality-of-life domains important to people with mental health conditions, which were independently found to be consistent with the CHIME framework and highlighted the additional 
importance of well-being/ill-being and physical health. The ReQoL also addresses a need to provide a quality-of-life measure for people with mental health conditions following criticism of the appropriateness of the existing generic measures (e.g. EuroQol-5D and Short Form-6D) in these conditions. In addition, the ReQoL has the potential to be used in the economic evaluation of mental health services.

\section{Routine use in mental health services}

Much has been made of the potential of PROMs to improve care and decision-making in the NHS, ${ }^{2}$ but the collection and analysis of routine data remains an important challenge. Importantly, the ReQoL is short ( 10 or 20 self-report items), which may help minimise the time and costs of collection. Using the ReQoL alongside a patient- reported experience measure; an indicator of the achievement of socially valued goals such as secure housing and valued work; and the achievement of individual recovery goals may help to provide a more complete picture of the quality and outcomes of mental health services.

\section{Conclusions}

It appears to be uncontroversial to say that we should judge the quality of our health services by whether it helps the people who use them. Nevertheless, the use of routine outcomes for people with mental health conditions that use mental health services has, as yet, not focused sufficiently on the patient's perspective. To improve this situation, service users and carers need to be involved in the entire process of developing, testing and measuring outcomes. This means listening effectively to the patient voice by developing a co-productive approach; developing a skilled and well-supported service-user research workforce; developing relevant and well-validated measures and using them appropriately. This cannot be done in isolation and requires a change in UK National Health Service and research culture from one that prioritises technical and professional expertise and a solely evidencebased approach, to one that gives equity to the value of expertise derived though lived experience and the coming together of evidence- and values-based approaches.

Jed Boardman, PhD, FRCPsych, King's College London Institute of Psychiatry,

Psychology \& Neuroscience, De Crespigny Park, London SE5 8AF, UK.

Email: jed.boardman@centreformentalhealth.org.uk

First Received 21 September 2017, accepted 3 October 2017

\section{References}

1 Devlin NJ, Appleby J. Getting the Most Out of PROMs. Putting Health Outcomes at the Heart of NHS Decision-Making. King's Fund, 2010.

2 Perkins $\mathrm{R}$. What constitutes success? The relative priority of service users' a clinicians' views of mental health services. Br J Psychiatry 2001; 179 : 9-10.

3 Nelson EC, Eftimovska E, Lind C, Hager A, Wasson JH, Lindblad S. Patient reported outcome measures in practice. Br Med J 2015; 350: g7818.

4 Crawford MJ, Robotham D, Thana L, Patterson S, Weaver T, Barber R, et al. Selecting outcome measures in mental health: the views of service users. J Ment Health 2011; 20: 336-46.

5 Trujols J, Portella MJ, Iraurgi I, Campins MJ, Siñol N, Pérez De Los Cobos J. Patient-reported outcome measures: are they patient-generated, patientcentred or patient-valued? J Ment Health 2013; 22: 555-62.

6 Rose D, Evans J, Sweeney A, Wykes T. A model for developing outcome measures from the perspectives of mental health service users. Int Rev Psychiatry 2011; 23: 41-6.

7 Patterson S, Trite J, Weaver T. Activity and views of service users involved in mental health research: UK survey. Br J Psychiatry 2014; 205: 68-75.

8 Ennis L, Wykes T. Impact of patient involvement in mental health research: longitudinal study. Br J Psychiatry 2013; 203: 381-6.

9 Keetharuth AD, Brazier J, Connell J, Bjorner JB, Carlton J, Buck ET, et al. Recovering Quality of Life (ReQoL): a new generic self-reported outcome measure for use with people experiencing mental health difficulties. Br J Psychiatry 2018; 212: 42-49.

10 Leamy M, Bird V, Le Boutillier C, Williams J, Slade M. Conceptual framework for personal recovery in mental health: systematic review and narrative synthesis. Br J Psychiatry 2011; 199: 445-52.

11. Shanks V, Williams J, Leamy M, Bird VJ, Le Boutillier C, Slade M Measures of personal recovery: a systematic review. Psychiatric Serv 2013; 64: 974-80. 